\title{
GEOMETRY OF MIXED STATES AND DEGENERACY STRUCTURE OF GEOMETRIC PHASES FOR MULTI-LEVEL QUANTUM SYSTEMS. A UNITARY GROUP APPROACH
}

\author{
E. ERCOLESSI, ${ }^{* \dagger}$ G. MARMO ${ }^{\ddagger}$ and G. MORANDI ${ }^{\dagger}$ \\ 'Dipartimento di Fisica, Universita' di Bologna, INFN and INFM, \\ Viale Berti-Pichat 6/2, 40127 Bologna, Italy \\ and \\ $\ddagger$ Dipartimento di Scienze Fisiche, Universita’ di Napoli Federico II and INFN, \\ Via Cinzia, 80126 Napoli, Italy \\ *Elisa.Ercolessi@bo.infn.it \\ N. MUKUNDA \\ Centre for Theoretical Studies and Department of Physics, \\ Indian Institute of Science, Bangalore 560 012, India \\ and \\ Jawaharlal Nehru Centre for Advanced Scientific Research, \\ Bangalore 560 064, India
}

\begin{abstract}
We analyze the geometric aspects of unitary evolution of general states for a multilevel quantum system by exploiting the structure of coadjoint orbits in the unitary group Lie algebra. Using the same methods in the case of SU(3) we study the effect of degeneracies on geometric phases for three-level systems. This is shown to lead to a highly nontrivial generalization of the result for two-level systems in which degeneracy results in a "monopole" structure in parameter space. The rich structures that arise are related to the geometry of adjoint orbits in SU(3). The limiting case of a two-level degeneracy in a three-level system is shown to lead to the known monopole structure.
\end{abstract}

\section{Introduction}

The original discovery and development of the geometric phase ideas were in the context of cyclic evolution within the adiabatic theorem of quantum mechanics (ATQM). ${ }^{7}$ In this treatment the adiabatic time dependence of the Hamiltonian operator was supposed to arise from its dependence on classical external parameters which in turn were taken to be slowly varying functions of time. Thus the quantum geometric phase was associated with a closed circuit in the external parameter space rather than in the Hilbert or ray space of the quantum system. 
Subsequent work has shown that the geometric phase can be viewed as belonging intrinsically to the ray space of the quantum system, and no reference needs to be made to the space of classical external parameters. ${ }^{25,1}$ The geometric phase idea has been applied also in the study of dissipative quantum-mechanical and classical dynamical systems. ${ }^{19}$ However the original picture shows its usefulness in certain physical situations and should therefore be retained. In particular the parameter space is most appropriate for studying the effects of degeneracies in the eigenvalue spectrum of the quantum Hamiltonian. In his original work, ${ }^{7}$ Berry studied the most important practical case of two-level degeneracy. Near such a point in parameter space, with no essential loss of generality one can suppose that there are generically three independent real parameters in the Hamiltonian. In accordance with the von Neumann-Wigner theorem it requires the vanishing of all three parameters to produce a degeneracy of levels in the Hamiltonian. In this threedimensional picture the point of degeneracy appears as a "magnetic monopole" (in parameter, not in physical space), and this singular point structure dominates the geometric phases for closed circuits in its vicinity.

The aim of this paper is to discuss some general geometric aspects of the unitary evolution of a quantum system with a finite number of levels, specializing later in a detailed study of the generic features of a three-level system, which would be the next level of complication in the quantum mechanical sense after two-level systems. For this problem, when degeneracies occur, the earlier "magnetic monopole" in the geometric phase discussion gets replaced by a far richer singularity structure in an eight-dimensional parameter space, and essentially new geometric as well as algebraic features arise. The situation hardly looks like a "monopole" any more. The basic reason is that while the treatment of two-level degeneracy involves the group $\mathrm{SU}(2)$ and its (real three-dimensional) adjoint representation, with three active levels one has to work with the group $\mathrm{SU}(3)$ and its (real eight-dimensional) adjoint representation. We make contact here with some previous work on $\mathrm{SU}(3),{ }^{2,3,20}$ putting however more emphasis on the geometric aspects involved in the analysis. The more general framework in which we study the occurrence of geometric phases and that is presented here can be of some relevance to studies and experiments on quantum and/or optical systems with a finite number of degrees of freedom that can be described on finite-dimensional Hilbert spaces, such as those occurring in many-channel (and three-channel in particular) optical interferometry, ${ }^{22,24,23}$ quantum computing ${ }^{27-29,12}$ or the study of entangled states ${ }^{18,28,16,21,14,6}$ of simple composite systems.

The material of the paper is organized as follows. In Sec. 2 we discuss some general features of a generic $n$-level quantum system as described, in the von Neumann picture, in the space of mixed states (or density matrices) and analyze the structure of the orbits under the action of the appropriate unitary group, i.e. $U(n)$. In Sec. 3 we collect the basic features of a Hermitian three-level Hamiltonian, its eigenvalues and eigenvectors and the regions of double and triple degeneracy in parameter space. We make use of the defining and adjoint representations of $\mathrm{SU}(3)$ 
and whenever possible bring in the $\mathrm{SU}(3)$ invariants of the problem. In Sec. 4 we discuss briefly the geometric properties of adjoint orbits in the Lie algebra of $\mathrm{SU}(3)$. Section 5 deals with the three independent geometric phase two-forms, relates them to the symplectic structures on the orbits, and develops a "sum rule" for them. Section 6 analyzes these two-forms from the point of view of their $\mathrm{SU}(3)$ transformation behavior and shows that there are three independent tensor contributions belonging respectively to the octet $(\underline{8})$, decouplet $(\underline{10})$ and antidecouplet $\left(\underline{10}{ }^{\star}\right)$ irreducible representations of $\mathrm{SU}(3)$. The details of recombining these three parts and reconstituting the complete two-forms are given. Section 7 studies the limiting case of a two-level degeneracy in a three-level system and traces in detail the emergence of the more familiar "monopole" structure as a special case in the present formalism. Section 8 contains some concluding remarks, and in the appendices some relevant materials related to $\mathrm{SU}(3)$ and its irreducible tensors, as needed here, are collected.

\section{General Aspects for $n$-Level Systems}

Many aspects of quantum dynamical systems are described on the space of mixed states (also called density matrices) where the evolution is ruled by the von Neumann equation:

$$
i \hbar \frac{d \rho}{d t}=[H, \rho]
$$

where $\rho$ describes the mixed state and $H$ is the (time-independent) Hamiltonian of the system. The von Neumann equation may be obtained from the Schrödinger equation:

$$
i \hbar \frac{d}{d t}|\psi\rangle=H|\psi\rangle
$$

with $\rho$ being defined as

$$
\rho=\sum_{j} c_{j}\left|\psi_{j}\right\rangle\left\langle\psi_{j}\right|, \quad c_{j} \in \mathbf{R}^{+}
$$

and the condition: $\operatorname{Tr} \rho<+\infty$.

The dynamical evolution given by integrating the von Neumann equation has the form:

$$
\rho(t)=\exp \left[-\frac{i H t}{\hbar}\right] \rho(0) \exp \left[\frac{i H t}{\hbar}\right]
$$

i.e. it is a conjugation in the space of mixed states.

In many physical situations (e.g. in many-channel interferometry and quantum computing $^{28,29,12}$ we deal actually with finite-level quantum systems, in which case the Hilbert space of states is given by $\mathcal{H}=\mathbf{C}^{n}$ for some $n$, and the group of unitary transformations is the unitary group $U(n)$. The orbits of the group, acting on the space of mixed states, will determine the carrier spaces of the dynamical evolution according to the von Neumann equation, i.e. each initial condition will select an 
orbit to which it belongs and the evolved state will remain in the same orbit. If instead a dissipative term is added on the r.h.s. of the von Neumann equation, in general the evolution will not be unitary anymore and the initial state will be carried from one orbit to another depending on the specific dissipation mechanism. These remarks point to the fact that the total space of the orbits may become relevant when dissipation occurs.

Here we are not going however to deal with particular dynamical systems but would rather like to concentrate our attention on the "carrier spaces," i.e. we are emphasizing the kinematical rather than the dynamical aspects. In particular we would like to consider the space of mixed states (convex combinations of pure states) and study how they are "partitioned" into orbits of $U(n)$ under conjugation. Eventually, in paving the way for the comparison of the von Neumann and the Schrödinger equations we will consider the connection one-form that is usually associated with the "geometric" or "Berry" phase.

The basic idea of our approach consists of embedding the space of mixed states in the space of all Hermitian matrices, i.e. instead of considering only convex combinations of pure states we will enlarge to combinations with arbitrary real coefficients. Hereafter we will multiply them by the imaginary unit $i$ so that they will define the Lie algebra $u(n)$ of the unitary group. With this extension our analysis will reduce to the analysis of the adjoint (or coadjoint) orbits of $U(n)$ on its Lie algebra.

The structures available on the space of matrices will allow us to consider bilinear products that are "inner" in $u(n)$, and this will provide us with computational tools making the analysis of the orbits more manageable. In the next sections more detailed computations will be carried out for a three-level quantum system, i.e. for $\mathrm{U}(3)$. In this particular context we will also exhibit the connection one-form or "Berry connection."

\subsection{Algebraic structures on the enlarged space of mixed states}

Given $\mathcal{H}=\mathbf{C}^{n}$, we consider an orthonormal basis: $\left\{\left|\psi_{i}\right\rangle\right\}_{i=1}^{n},\left\langle\psi_{i} \mid \psi_{j}\right\rangle=\delta_{i j}$, and construct a basis $\left\{E_{a b}, E_{a b}^{\prime}\right\}$, in the space of Hermitian matrices as

$$
\begin{aligned}
& E_{a a}=\left|\psi_{a}\right\rangle\left\langle\psi_{a}\right|, \\
& E_{a b}=\left|\psi_{a}\right\rangle\left\langle\psi_{b}|+| \psi_{b}\right\rangle\left\langle\psi_{a}\right|, \quad a<b=1, \ldots, n
\end{aligned}
$$

and

$$
E_{a b}^{\prime}=i\left\{\left|\psi_{a}\right\rangle\left\langle\psi_{b}|-| \psi_{b}\right\rangle\left\langle\psi_{a}\right|\right\}, \quad a<b=1, \ldots, n
$$

Any Hermitian matrix $H$ can be written in the form:

$$
H=\xi^{a b} E_{a b}+\xi^{\prime a b} E_{a b}^{\prime}
$$

with real coefficients. 
In this real vector space we may consider two bilinear products, namely:

$$
H_{1} * H_{2}=\frac{1}{2}\left\{H_{1} H_{2}+H_{2} H_{1}\right\}
$$

which is commutative but not associative, and

$$
H_{1} \wedge H_{2}=i\left\{H_{1} H_{2}-H_{2} H_{1}\right\}
$$

which defines a Lie algebra structure. Also, a scalar product is inherited from that in $\mathcal{H}$, namely:

$$
H_{1} \cdot H_{2}=\operatorname{Tr}\left\{H_{1} H_{2}\right\}
$$

The specific way we have written our bilinear structures permits us to write down several identities coming from the "interaction" of these products. We list below some of them:

(i) The scalar product is invariant under conjugation:

$$
H_{1} \cdot H_{2}=U\left(H_{1}\right) \cdot U\left(H_{2}\right)
$$

where

$$
U(H)=U^{\dagger} H U, \quad U \in U(n) .
$$

(ii) If $H$ is generic, the set of powers $H^{0}, H^{1}, H^{2}, \ldots, H^{n-1}$ defines a maximal set of commuting Hermitian matrices. This generates the commutant of $H$, and it is Abelian. When $H$ is not generic, i.e. there are degeneracies in its spectrum, not all powers are independent, and the independent ones are in a number equal to the degree of the minimal polynomial associated with $H$. The commutant of $H$ will be no more Abelian and will have dimension greater than $n$.

(iii) Two Hermitian matrices will be in the same orbit iff they have the same characteristic polynomial. We recall that the characteristic polynomial $P(\lambda)$ is defined as

$$
P(\lambda)=\lambda^{n}+c_{1} \lambda^{n-1}+\cdots+c_{n}=(-1)^{n} \operatorname{Det}\{H-\lambda \mathbf{I}\},
$$

where

$$
c_{1}=-\operatorname{Tr} H, \quad c_{k}=-\left\{\operatorname{Tr}\left(H^{k}\right)+c_{1} \operatorname{Tr}\left(H^{k-1}\right)+\cdots+c_{k-1} \operatorname{Tr} H\right\}, \quad k \geq 2 .
$$

(iv) Two orbits will be of the same type iff their commutants (seen as subalgebras of $u(n))$ are isomorphic.

(v) Each orbit is a symplectic manifold and is symplectomorphic with $U(n) / U_{H}$, where $H$ is an element of the orbit and $U_{H}$ its stabilizer under conjugation, i.e.

$$
U_{H}=\left\{U \in U(n) \vdash U^{\dagger} H U=H\right\} .
$$


For a generic $H$ :

$$
U_{H}=\underbrace{\mathrm{U}(1) \times \mathrm{U}(1) \times \cdots \times \mathrm{U}(1)}_{n \text { times }} .
$$

At the other extreme, if $H$ corresponds to a pure state projection, $H=|\psi\rangle\langle\psi|$, then: $U_{H}=\mathrm{U}(1) \times U(n-1)$. The space of pure states is therefore given by

$$
\mathcal{P}(\mathcal{H})=\frac{U(n)}{\mathrm{U}(1) \times U(n-1)} \approx \frac{\mathrm{SU}(n)}{\mathrm{U}(1) \times \mathrm{SU}(n-1)} .
$$

(vi) On each orbit there is a symplectic structure ${ }^{4,5}$ given by

$$
\omega_{H}=\operatorname{Tr}\left\{H d U^{\dagger} \wedge d U\right\},
$$

where $U \in U(n)$. The way $\omega_{H}$ has been written defines actually a two-form on the whole of $U(n)$, which is however degenerate. It is not difficult to show that the kernel of $\omega_{H}$ coincides with the Lie algebra of $U_{H}$. Therefore $\omega_{H}$ "descends to the quotient" and defines a nondegenerate two-form on the orbit.

(vii) On $U(n) \omega_{H}$ is exact, and is given by ${ }^{4,5}$

$$
\omega_{H}=d \operatorname{Tr}\left\{H U^{\dagger} d U\right\}=-\operatorname{Tr}\left\{H U^{\dagger} d U \wedge U^{\dagger} d U\right\} .
$$

The one-form $\operatorname{Tr}\left\{H U^{\dagger} d U\right\}$ however does not descend to the quotient, implying that on each orbit the symplectic form is closed and nondegenerate, but not exact.

We recall that if $G$ is any Lie group and $X_{A}, X_{B}$ are the left-invariant vector fields associated with $A, B$ in the Lie algebra $g$ of $G, \imath_{X_{A}}\left(U^{\dagger} d U\right)=A$ and $\imath_{X_{B}}\left(U^{\dagger} d U\right)=B$, then

$$
\begin{aligned}
\omega(H)\left(X_{A}, X_{B}\right)= & -\operatorname{Tr}\{H[A, B]\} \\
= & -\operatorname{Tr}\left\{H U^{\dagger} d U\left(X_{A}\right) U^{\dagger} d U\left(X_{B}\right)\right\} \\
& +\operatorname{Tr}\left\{H U^{\dagger} d U\left(X_{B}\right) U^{\dagger} d U\left(X_{A}\right)\right\}
\end{aligned}
$$

Then

$$
\omega(H)\left(X_{A}, X_{B}\right)=\operatorname{Tr}\{H[A, B]\}=\operatorname{Tr}\{[B, H] A\}=\operatorname{Tr}\{[H, A] B\}
$$

and it is clear that the kernel of $\omega$ coincides with the vector fields generated by the commutant of $H$. Going to the quotient we obtain the symplectic structures on the orbits. In the case of $\mathrm{SU}(3)$ an explicit expression in terms of the Euler angles is given in Refs. 11, 9 and 8.

\section{Structure of the Hamiltonian, Spectrum and Degeneracies for Three-Level Systems}

For a quantum system in which three (generically) distinct energy eigenvalues and eigenvectors play a dominant role and the other states may be neglected, the generic Hamiltonian is some Hermitian three-dimensional matrix. With no loss of generality 
and whenever needed, by subtracting an appropriate multiple of the identity, we may remove the trace of the matrix, so that at the point of triple degeneracy all three eigenvalues vanish. We recall that the set of all Hermitian $3 \times 3$ matrices defines the Lie algebra $\mathrm{u}(3)$ of the unitary group $\mathrm{U}(3)$, while the traceless ones define the Lie algebra $\mathrm{su}(3)$ of $\mathrm{SU}(3)$.

A basis of generators for $\mathrm{su}(3)$ is given by the (Hermitian and traceless) GellMann matrices ${ }^{15,13}$ of App. A.

A generic $3 \times 3$ Hermitian matrix can be uniquely represented in the basis of the Gell-Mann generators ${ }^{15}$ as

$$
H(\xi)=\xi_{0} \lambda_{0}+\frac{1}{2} \boldsymbol{\xi} \cdot \boldsymbol{\lambda},
$$

where $\lambda_{0}=\mathbf{I}$ is the identity matrix, $\xi=\left(\xi_{0}, \boldsymbol{\xi}\right), \xi_{0}=\frac{1}{3} \operatorname{Tr}\{H\}$ and $\boldsymbol{\xi}$ is an eightdimensional real vector, $\boldsymbol{\xi} \in \mathbf{R}^{8}$. Of course: $\xi_{0}=0$ for traceless matrices (and vice versa).

Under conjugation with $U \in \mathrm{SU}(3), H$ transforms as

$$
U H(\xi) U^{\dagger}=\xi_{0} \lambda_{0}+\frac{1}{2} \boldsymbol{\xi} \cdot U \boldsymbol{\lambda} U^{\dagger}=: \xi_{0} \lambda_{0}+\frac{1}{2} \boldsymbol{\xi}^{\prime} \cdot \boldsymbol{\lambda},
$$

where (see formula (A.8)):

$$
\xi_{r}^{\prime}=D_{r s}(U) \xi_{s} .
$$

The bilinear products discussed in Subsec. 2.1 induce binary operations among vectors in $\mathbf{R}^{8}$. If: $H_{1}=H\left(\xi^{1}\right), H_{2}=H\left(\xi^{2}\right)$, then

$$
H_{1} \wedge H_{2}=\left(\boldsymbol{\xi}^{1} \wedge \boldsymbol{\xi}^{2}\right) \cdot \boldsymbol{\lambda},
$$

where

$$
\left(\boldsymbol{\xi}^{1} \wedge \boldsymbol{\xi}^{2}\right)_{r}=-\frac{1}{2} f_{r s t} \xi_{s}^{1} \xi_{t}^{2}
$$

(ii) $\quad H_{1} * H_{2}=\frac{1}{3} \lambda_{0} \operatorname{Tr}\left\{H_{1} H_{2}\right\}+\frac{1}{2}\left\{\xi_{0}^{1} \boldsymbol{\xi}^{2}+\xi_{0}^{2} \boldsymbol{\xi}^{1}\right\} \cdot \boldsymbol{\lambda}+\frac{1}{4 \sqrt{3}}\left(\boldsymbol{\xi}^{1} * \boldsymbol{\xi}^{2}\right) \cdot \boldsymbol{\lambda}$,

where

$$
\left(\boldsymbol{\xi}^{1} * \boldsymbol{\xi}^{2}\right)_{r}=\sqrt{3} d_{r s t} \xi_{s}^{1} \xi_{t}^{2}
$$

and

$$
\operatorname{Tr}\left(H_{1} H_{2}\right)=3 \xi_{0}^{1} \xi_{0}^{2}+\frac{1}{2} \boldsymbol{\xi}^{1} \cdot \boldsymbol{\xi}^{2} .
$$

In particular, for traceless matrices:

$$
\operatorname{Tr}\left(H_{1} H_{2}\right)=\frac{1}{2} \boldsymbol{\xi}^{1} \cdot \boldsymbol{\xi}^{2}
$$

and

$$
H_{1} * H_{2}=\frac{1}{6}\left(\boldsymbol{\xi}^{1} \cdot \boldsymbol{\xi}^{2}\right) \lambda_{0}+\frac{1}{4 \sqrt{3}}\left(\boldsymbol{\xi}^{1} * \boldsymbol{\xi}^{2}\right) \cdot \boldsymbol{\lambda}
$$


and, if $H_{3}$ is another traceless Hermitian matrix:

$$
\operatorname{Tr}\left\{\left(H_{1} * H_{2}\right) H_{3}\right\}=\frac{1}{2} \operatorname{Tr}\left\{\left(H_{1} H_{2}+H_{2} H_{1}\right) H_{3}\right\}=\frac{1}{4 \sqrt{3}}\left(\boldsymbol{\xi}^{1} * \boldsymbol{\xi}^{2}\right) \cdot \boldsymbol{\xi}^{3} .
$$

Quite obviously then, both

$$
\boldsymbol{\xi} \cdot \boldsymbol{\xi}=2 \operatorname{Tr}\left\{H(\xi)^{2}\right\}
$$

and

$$
(\boldsymbol{\xi} * \boldsymbol{\xi}) \cdot \boldsymbol{\xi}=4 \sqrt{3} \operatorname{Tr}\left\{H(\xi)^{3}\right\} \equiv \sqrt{3} d_{r s t} \xi_{r} \xi_{s} \xi_{t}
$$

will be invariant under conjugation and will provide us with a quadratic and a cubic invariant respectively. ${ }^{3,20}$ A third (linear) invariant would be $\operatorname{Tr}\{H\}$ whenever $H$ is not traceless. Notice that $(\boldsymbol{\xi} * \boldsymbol{\xi}) \cdot \boldsymbol{\xi}$ is bounded both from above and from below, and indeed it can be proved that

$$
-|\boldsymbol{\xi}|^{3} \leq(\boldsymbol{\xi} * \boldsymbol{\xi}) \cdot \boldsymbol{\xi} \leq|\boldsymbol{\xi}|^{3},
$$

where, in an obvious notation: $|\boldsymbol{\xi}|=: \sqrt{\boldsymbol{\xi} \cdot \boldsymbol{\xi}}$. Remember also that

$$
\operatorname{det}(H(\xi))=\frac{1}{2 \sqrt{3}}(\boldsymbol{\xi} * \boldsymbol{\xi}) \cdot \boldsymbol{\xi} .
$$

The eigenvalues of $H(\xi)$ (for $\xi_{0}=0$ ) can be conveniently expressed as follows. ${ }^{3}$ For each given $\boldsymbol{\xi}$, we define first an angle $\phi$ in the range $[\pi / 6, \pi / 2]$ by

$$
(\boldsymbol{\xi} * \boldsymbol{\xi}) \cdot \boldsymbol{\xi}=-|\boldsymbol{\xi}|^{3} \sin (3 \phi) .
$$

Given the limitations on $(\boldsymbol{\xi} * \boldsymbol{\xi}) \cdot \boldsymbol{\xi}$ and the range specified for $\phi$, the angle is uniquely determined by $\boldsymbol{\xi}$ (except for $\boldsymbol{\xi}=0$, of course) and is by construction an $\mathrm{SU}(3)$-invariant. The eigenvalues of $H(\xi)$, written as $E_{a}(\boldsymbol{\xi}), a=1,2,3$, are given in nonincreasing order by

$$
\begin{aligned}
& E_{1}(\boldsymbol{\xi})=\frac{|\boldsymbol{\xi}|}{\sqrt{3}} \sin \phi, \\
& E_{2}(\boldsymbol{\xi})=\frac{|\boldsymbol{\xi}|}{\sqrt{3}} \sin \left(\phi+\frac{2 \pi}{3}\right), \\
& E_{3}(\boldsymbol{\xi})=\frac{|\boldsymbol{\xi}|}{\sqrt{3}} \sin \left(\phi+\frac{4 \pi}{3}\right), \\
& E_{1}(\boldsymbol{\xi}) \geq E_{2}(\boldsymbol{\xi}) \geq E_{3}(\boldsymbol{\xi}), \quad E_{1}(\boldsymbol{\xi})+E_{2}(\boldsymbol{\xi})+E_{3}(\boldsymbol{\xi})=0 .
\end{aligned}
$$

The successive energy differences have the simple forms:

$$
E_{12}(\boldsymbol{\xi})=E_{1}(\boldsymbol{\xi})-E_{2}(\boldsymbol{\xi})=|\boldsymbol{\xi}| \sin \left(\phi-\frac{\pi}{6}\right)
$$

and

$$
E_{23}(\boldsymbol{\xi})=E_{2}(\boldsymbol{\xi})-E_{3}(\boldsymbol{\xi})=|\boldsymbol{\xi}| \cos \phi .
$$

We will sometimes write simply $E_{a}, E_{a b}$, omitting explicit mention of $|\boldsymbol{\xi}|$. 
It is easy to check that double degeneracies occur only for $\phi$ at the extremes of the interval of definition, and precisely that

$$
\phi=\frac{\pi}{6} \Rightarrow E_{12}=0
$$

the upper double degeneracy,

$$
\phi=\frac{\pi}{2} \Rightarrow E_{23}=0
$$

the lower double degeneracy, while for $\phi \in(\pi / 6, \pi / 2)$ the Hamiltonian is nondegenerate, i.e. $E_{12}, E_{23}>0$, while triple degeneracy occurs only for $\boldsymbol{\xi}=0$.

Including dilations, the regions of double degeneracy are two distinct nonoverlapping five-dimensional regions in $\mathbf{R}^{8}-\{\mathbf{0}\}$, each one comprising a singular fourparameter family of directions. Denoting these regions by $\Sigma_{12}$ and $\Sigma_{23}$ respectively:

$$
\Sigma_{12}=\left\{\boldsymbol{\xi} \in \mathbf{R}^{8}-\{\mathbf{0}\}:(\boldsymbol{\xi} * \boldsymbol{\xi}) \cdot \boldsymbol{\xi}=-|\boldsymbol{\xi}|^{3}\right\}
$$

corresponding to $E_{12}=0, E_{23}>0$, and:

$$
\Sigma_{23}=\left\{\boldsymbol{\xi} \in \mathbf{R}^{8}-\{\mathbf{0}\}:(\boldsymbol{\xi} * \boldsymbol{\xi}) \cdot \boldsymbol{\xi}=+|\boldsymbol{\xi}|^{3}\right\}
$$

corresponding to $E_{12}>0, E_{23}=0$.

We shall mostly deal with the generic, nondegenerate situation. Let us denote the three orthonormal eigenvectors of $H(\xi)$ by $|a ; \boldsymbol{\xi}\rangle, a=1,2,3$ :

$$
H(\xi)|a ; \boldsymbol{\xi}\rangle=E_{a}(\boldsymbol{\xi})|a ; \boldsymbol{\xi}\rangle, \quad\langle a ; \boldsymbol{\xi} \mid b ; \boldsymbol{\xi}\rangle=\delta_{a b}
$$

The overall phases of these eigenvectors are at the moment free. The standard orthonormal basis for the complex three-dimensional space $\mathbf{C}^{3}$ is written simply as $|a\rangle$, with

$$
|1\rangle=\left[\begin{array}{l}
1 \\
0 \\
0
\end{array}\right], \quad|2\rangle=\left[\begin{array}{l}
0 \\
1 \\
0
\end{array}\right], \quad|3\rangle=\left[\begin{array}{l}
0 \\
0 \\
1
\end{array}\right] .
$$

Then the matrix $A(\boldsymbol{\xi})$ defined by

$$
A_{a b}(\boldsymbol{\xi})=:\langle a \mid b ; \boldsymbol{\xi}\rangle
$$

is unitary, $A \in \mathrm{U}(3)$, and relates the two bases:

$$
|a ; \boldsymbol{\xi}\rangle=A_{b a}(\boldsymbol{\xi})|b\rangle
$$

(sums over repeated indices being understood). We will assume that the phases of the three eigenvectors $|a ; \boldsymbol{\xi}\rangle$ are adjusted in such a way that actually $A(\boldsymbol{\xi}) \in \mathrm{SU}(3)$. This still leaves two free phases in $A(\boldsymbol{\xi})$.

It is clear that conjugation of $H(\boldsymbol{\xi})$ by $A(\boldsymbol{\xi})$ reduces it to diagonal form. In this process $\boldsymbol{\xi}$ gets transformed to a $\boldsymbol{\xi}^{(0)}$ of which only the third and eighth components are nonzero and moreover, in agreement with the ordering of the eigenvalues, the 
diagonal entries of $H\left(\boldsymbol{\xi}^{(0)}\right)$ are nonincreasing. We shall call the resulting unique $\boldsymbol{\xi}^{(0)}$ the "rest frame" form ${ }^{20}$ of $\boldsymbol{\xi}$. Therefore

$$
A(\boldsymbol{\xi})^{\dagger} H(\boldsymbol{\xi}) A(\boldsymbol{\xi})=H\left(\boldsymbol{\xi}^{(0)}\right), \quad \boldsymbol{\xi}^{(0)}=\left(0,0, \xi_{3}^{(0)}, 0,0,0,0, \xi_{8}^{(0)}\right)
$$

with $\xi_{8}^{(0)} \geq \frac{\xi_{3}^{(0)}}{\sqrt{3}} \geq 0$ and

$$
\begin{aligned}
& E_{1}=\frac{1}{2}\left(\xi_{3}^{(0)}+\frac{1}{\sqrt{3}} \xi_{8}^{(0)}\right), \\
& E_{2}=\frac{1}{2}\left(-\xi_{3}^{(0)}+\frac{1}{\sqrt{3}} \xi_{8}^{(0)}\right), \\
& E_{3}=-\frac{1}{\sqrt{3}} \xi_{8}^{(0)} .
\end{aligned}
$$

Moreover

$$
\begin{aligned}
\boldsymbol{\xi}^{(0)} * \boldsymbol{\xi}^{(0)} & =\left(0,0,2 \xi_{3}^{(0)} \xi_{8}^{(0)}, 0,0,0,0, \xi_{3}^{(0) 2}-\xi_{8}^{(0) 2}\right) \\
|\boldsymbol{\xi}|^{2} & =\xi_{3}^{(0) 2}+\xi_{8}^{(0) 2}
\end{aligned}
$$

and

$$
\boldsymbol{\xi}^{(0)} * \boldsymbol{\xi}^{(0)} \cdot \boldsymbol{\xi}^{(0)}=\xi_{8}^{(0)}\left(3 \xi_{3}^{(0) 2}-\xi_{8}^{(0) 2}\right) .
$$

Notice that all this implies

$$
\begin{aligned}
& \xi_{3}^{(0)}=0 \Leftrightarrow \boldsymbol{\xi} * \boldsymbol{\xi} \cdot \boldsymbol{\xi}=-|\boldsymbol{\xi}|^{3} \Leftrightarrow E_{12}=0, \\
& \xi_{3}^{(0)}=\sqrt{3} \xi_{8}^{(0)} \Leftrightarrow \boldsymbol{\xi} * \boldsymbol{\xi} \cdot \boldsymbol{\xi}=|\boldsymbol{\xi}|^{3} \Leftrightarrow E_{23}=0 .
\end{aligned}
$$

It should be noted that, while $\boldsymbol{\xi}^{(0)} * \boldsymbol{\xi}^{(0)} \cdot \boldsymbol{\lambda}$ is diagonal, in general $\boldsymbol{\xi}^{(0)} * \boldsymbol{\xi}^{(0)}$ is not in rest frame form.

For general $\boldsymbol{\xi}$, the phase freedom in the eigenvectors of $H(\xi)$, even with the condition $A(\boldsymbol{\xi}) \in \mathrm{SU}(3)$ corresponds to the fact that $\boldsymbol{\xi}^{(0)}$ has a nontrivial stability subgroup $\mathrm{U}(1) \times \mathrm{U}(1) \subset \mathrm{SU}(3)$, the torus subgroup. Therefore $A(\boldsymbol{\xi})$ remains undefined up to such an element on the right. This is evident if we rewrite the relation between $H(\xi)$ and $H\left(\xi^{(0)}\right)$ as

$$
H(\xi)=A(\boldsymbol{\xi}) H\left(\xi^{(0)}\right) A(\boldsymbol{\xi})^{\dagger} .
$$

We shall often refer to this freedom on the right in the choice of $A(\boldsymbol{\xi})$ for each $\boldsymbol{\xi} \in \mathbf{R}^{8}-\{\mathbf{0}\}$.

\section{Remark}

If, for $\boldsymbol{\xi} \neq 0$ (i.e. on $\mathbf{R}^{8}-\{\mathbf{0}\}$ ) we normalize $\boldsymbol{\xi}$ by requiring, e.g. $\boldsymbol{\xi} \cdot \boldsymbol{\xi}=1$ or, equivalently, by quotienting w.r.t. the dilations in $\mathbf{R}^{8}-\{0\}$, we obtain the sphere $S^{7}$ of normalized vectors. By quotienting further w.r.t. the U(1) isotropy group 
(the "phase isotropy subgroup") of the vectors, we obtain eventually the projective plane $\mathrm{CP}^{3}$. All in all, we have the sequence of fibrations:

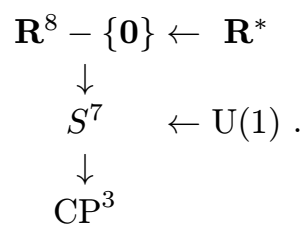

This implies that a study of the geometry of the generic orbits may turn out to be relevant for the study of the geometry of entangled states as well. This comes about because the manifold of pure states for a composite system of two two-level systems (such as photons or spin-1/2 particles) is $\mathbf{C}^{4}$ and the associated projective space is $\mathrm{CP}^{3}$. Product pure states are given by the four-dimensional $\mathrm{CP}^{1} \times \mathrm{CP}^{1}$ and, in general, the pure states of a composite $2 \times 2$ quantum system span $\mathrm{U}(4) / \mathrm{U}(3) \times$ $\mathrm{U}(1) \approx \mathrm{CP}^{3} \rightleftharpoons \mathbf{R}^{8}-\{\mathbf{0}\} / \mathbf{R}^{*} \times \mathrm{U}(\mathbf{1})$, which is diffeomorphic to the generic coadjoint orbits of $\mathrm{SU}(3)$.

As stated in Sec. 2, the coadjoint orbits are symplectic manifolds, the symplectic form being given by Eq. (19). $\boldsymbol{\omega}_{H}^{3}$ will be a volume-form and will define a differentiable measure on the orbit. When pulled back to $S^{7}$, and identifying for short $\boldsymbol{\omega}_{H}^{3}$ with its pull-back, $\boldsymbol{\omega}_{H}^{3} \wedge d \theta$ will be a volume-form on $S^{7}$ and, similarly, $\boldsymbol{\omega}_{H}^{3} \wedge d \theta \wedge d r / r$ will be a volume-form on $\mathbf{R}^{8}-\{\mathbf{0}\}$, the latter providing a $\left(\right.$ Bures $\left.^{26,10}\right)$ measure on the space of density matrices.

Finally, the orbits can also be equipped with a metric. Indeed, if $\left\{H_{i}\right\}$ is a basis in the Lie algebra $u(n)$ of $U(n)$ (e.g. the one given in Eqs. (5)-(7) we can also define a left-invariant metric $g$ on $U(n)$ as

$$
g=\delta^{i j}\left(\operatorname{Tr} H_{i} U^{\dagger} d U\right) \otimes\left(\operatorname{Tr} H_{j} U^{\dagger} d U\right)
$$

and similarly for a right-invariant one, or a bi-invariant metric as

$$
\widetilde{g}=\operatorname{Tr}\left(U^{\dagger} d U \otimes U^{\dagger} d U\right)
$$

and on each orbit we have a metric like

$$
\operatorname{Tr}\left(\left[H, U^{\dagger} d U\right] \otimes\left[H, U^{\dagger} d U\right]\right)
$$

or

$$
\operatorname{Tr}\left(\left[H, d U U^{\dagger}\right] \otimes\left[H, d U U^{\dagger}\right]\right)
$$

according to which action has been chosen to define the quotient.

\section{Some Geometry of the Adjoint Orbits of SU(3)}

As already stated in Sec. 2, we will be interested in the orbits of SU(3) in the space of the traceless Hermitian matrices or in those of $\mathrm{U}(3)$ in the space of all Hermitian matrices. 
To make contact with the previous section, we recall that each orbit is characterized uniquely by the isotropy group of any one of its elements (the isotropy groups for different elements being conjugate subgroups of $\mathrm{SU}(3)$ or $\mathrm{U}(3)$ as the case may be), and that the latter is generated by the commutant in the way that has been discussed in Sec. 2 and that will be rephrased here in the specific context of $\mathrm{SU}(3)$ (or $\mathrm{U}(3))$.

If $H$ is generic, the isotropy group is generated by $H, H^{2}$ and $H^{3}$ as

$$
\left\{e^{i s_{1} H}, e^{i s_{2} H^{2}}, e^{i s_{3} H^{3}}\right\}=\mathrm{U}(1) \times \mathrm{U}(1) \times \mathrm{U}(1)
$$

and the invariants characterizing the orbits in $\mathrm{U}(3)$ will be $\operatorname{Tr}(H), \operatorname{Tr}\left(H^{2}\right)$ and $\operatorname{Tr}\left(H^{3}\right)$. If we restrict to traceless matrices we are left with $\operatorname{Tr}\left(H^{2}\right)$ and $\operatorname{Tr}\left(H^{3}\right)$ and, according to the discussion of Sec. 2 , we can replace $\operatorname{Tr}\left(H^{3}\right)$ with $\operatorname{Det}(H)$. For more details see Ref. 17. Also, the isotropy group in $\mathrm{SU}(3)$ will be $\mathrm{U}(1) \times \mathrm{U}(1)$ (compare the discussion of the previous section). Explicit expressions for the invariants in terms of the vector $\boldsymbol{\xi}$ have been given before and will not be reproduced here.

If $H$ is not generic and has a single doubly degenerate eigenvalue the isotropy group will be $\mathrm{U}(2) \subset \mathrm{SU}(3)$ ( or $\mathrm{U}(2) \times \mathrm{U}(1)$ in $\mathrm{U}(3)$ ). Finally, when $H$ has a triply degenerate eigenvalue the isotropy group will become the whole of $\mathrm{SU}(3)$ (or $\mathrm{U}(3)$ ).

For any group $G$ and for any given $H \in \underline{G}$, the Lie algebra of $G$, the orbit through $H$ will be diffeomorphic with the coset space $G / K_{H}$, with $K_{H}$ the isotropy group of $H$. We can also identify the orbit by taking $H$ in its rest frame. With $H$ fixed in this manner, we can write the symplectic structure on this coset space as

$$
\omega(H)=d\left\{\operatorname{Tr} H s^{-1} d s\right\}, \quad s \in G
$$

(if $G=\mathrm{U}(3)$ or $\mathrm{SU}(3), s^{-1}=s^{\dagger}$ ) or, using $d s^{-1}=-s^{-1} d s s^{-1}$ :

$$
\omega(H)=-\operatorname{Tr} H\left\{s^{-1} d s \wedge s^{-1} d s\right\} .
$$

Generic orbits will be defined in $\mathbf{R}^{8}$ by the algebraic equations:

$$
\begin{gathered}
\boldsymbol{\xi} \cdot \boldsymbol{\xi}=\xi^{2}=\text { const }=c_{1}, \\
\boldsymbol{\xi} * \boldsymbol{\xi} \cdot \boldsymbol{\xi}=\text { const }=c_{2} \neq \pm \xi^{3} .
\end{gathered}
$$

The intersection of these algebraic varieties yields the (generic) symplectic orbit which is six-dimensional and is diffeomorphic with $\mathrm{SU}(3) / \mathrm{U}(1) \times \mathrm{U}(1) \approx \mathrm{U}(3) /$ $\mathrm{U}(1) \times \mathrm{U}(1) \times \mathrm{U}(1) \approx \mathrm{CP}^{3}$.

Four-dimensional exceptional orbits are defined by intersecting $\xi^{2}=c_{1}$ with the subspaces $\Sigma_{12}$ or $\Sigma_{23}$ defined in Sec. 3. The intersections are diffeomorphic with $\mathrm{SU}(3) / \mathrm{U}(2)$.

\section{Remarks}

(1) Assuming that $H$ be traceless does not seem to play a relevant role in this context, and indeed the commutant of $H$ does not change if we add or subtract from $H$ any multiple of the identity. 
(2) Given a doubly degenerate eigenvalue for $H$, say

$$
H=\lambda\{|1\rangle\langle 1|+| 2\rangle\langle 2|\}+\mu|3\rangle\langle 3|, \quad \lambda \neq \mu
$$

we may add $-\lambda \mathbf{I}$ to $H$ to get

$$
(\mu-\lambda)^{-1}(H-\lambda \mathbf{I})=|3\rangle\langle 3| .
$$

Therefore the symplectic orbit through a pure state is diffeomorphic to the symplectic orbit through a matrix with a doubly degenerate eigenvalue.

(3) The foliation of $\mathrm{U}(3)$ defined by the level sets of the function:

$$
i \operatorname{Tr}: u(3) \rightarrow \mathbf{R}
$$

include orbits of the coadjoint action. By considering the intersection with $(i \operatorname{Tr})^{-1}(0)$ we find orbits of $\mathrm{SU}(3)$ in the dual algebra of $\mathrm{su}(3)$.

(4) Starting from the Hilbert space $\mathcal{H}$ we may consider the projection: $\pi: \mathcal{H} \backslash\{0\} \rightarrow$ $\mathcal{P}(\mathcal{H})$. In Dirac's notation

$$
\pi:|\psi\rangle \mapsto \frac{|\psi\rangle\langle\psi|}{\langle\psi \mid \psi\rangle} .
$$

Notice that in this way $\mathcal{H} \backslash\{0\}$ becomes a principal C-bundle over $\mathcal{P}(\mathcal{H})$. Moreover

$$
\pi(|\psi\rangle+|\phi\rangle) \propto|\psi\rangle\langle\psi|+| \phi\rangle\langle\phi|+(|\psi\rangle\langle\phi|+| \phi\rangle\langle\psi|)
$$

and

$$
\pi(|\psi\rangle+i|\phi\rangle) \propto|\psi\rangle\langle\psi|+| \phi\rangle\langle\phi|-i(|\psi\rangle\langle\phi|-| \phi\rangle\langle\psi|)
$$

so under projection we generate both $|\psi\rangle\langle\phi|+| \phi\rangle\langle\psi|$ and $i(|\psi\rangle\langle\phi|-| \phi\rangle\langle\psi|)$. Hence all Hermitian matrices can be written as combinations over the reals of elements in $\mathcal{P}(\mathcal{H})$. Appropriate restrictions on the coefficients will select the space of density matrices.

\section{Geometric Phase Two-Forms and U(1) Connections over Coadjoint Orbits}

After this general discussion of the structure of the space of all (pure and mixed) states, we turn to the geometric phase problem for three-level systems.

Following the adiabatic theorem of quantum mechanics we can imagine carrying any one of the three nondegenerate eigenvectors $|a ; \xi\rangle$ of the (generic) Hamiltonian $H(\xi)$ along a closed path $\mathcal{C} \subset \mathbf{R}^{8}$, avoiding the origin and the subsets $\Sigma_{12}$ and $\Sigma_{23}$, and we can then ask how much geometrical phase it has accumulated. From a physical point of view we would consider circuits "close" to the origin $\boldsymbol{\xi}=0$. Thus we are concerned with three different geometrical phases $\phi^{(a)}(\mathcal{C}), a=1,2,3$, each one associated with a given eigenvector. Notice that in the case of a two-level system, when the relevant group is $\mathrm{SU}(2)$, one has also two in principle different geometrical phases. However, as they differ by a constant $(4 \pi)$ and by a sign, one 
is accustomed to speak of a single "Berry phase" in that context. Here we will be forced to deal with three phases. We will discuss in what follows some more general "sum rule" among them.

It is known that the above geometric phases can be computed as integrals over any two-dimensional surface bounded by $\mathcal{C}$ in $\mathbf{R}^{8}-\left\{\{0\} \cup \Sigma_{12} \cup \Sigma_{23}\right\}$ of corresponding two-forms $V^{(a)}(\xi)$. Such forms are the curvature forms associated with the corresponding "Berry phase" connection one-forms. The general expression for these two-forms describing the "flux" of the geometric phase in parameter space is ${ }^{7}$

$$
\begin{aligned}
V^{(a)}(\xi) & =\operatorname{Im} \sum_{b \neq a} \frac{\langle a ; \xi|d H(\xi)| b ; \xi\rangle \wedge\langle b ; \xi|d H(\xi)| a ; \xi\rangle}{E_{a b}(\xi)^{2}} \\
& =\frac{1}{2} V_{r s}^{(a)}(\xi) d \xi_{r} \wedge d \xi_{s},
\end{aligned}
$$

where

$$
V_{r s}^{(a)}(\xi)=\frac{1}{4} \operatorname{Im} \sum_{b \neq a} \frac{\left\langle a ; \xi\left|\lambda_{r}\right| b ; \xi\right\rangle\left\langle b ; \xi\left|\lambda_{s}\right| a ; \xi\right\rangle-\{r \leftrightarrow s\}}{E_{a b}(\xi)^{2}}
$$

Before proceeding with the analysis of the $V^{(a)}$ 's, let us pause a moment and see how they arise in the geometric context of the (co)adjoint orbits of SU(3) in the space of Hermitian matrices.

Let then $H=H(\xi)$ be a Hermitian matrix (we will not need to impose here conditions on its trace), and consider the eigenvalue problem:

$$
H(\xi)|a ; \xi\rangle=E_{a}(\xi)|a ; \xi\rangle
$$

along with the orbit of $H(\xi)$ under conjugation. By using, as a shorthand for Eq. (23), $H(\xi)=\frac{1}{2} \xi \cdot \lambda$ we may consider the particular $\xi^{(0)}$ such that $H\left(\xi^{(0)}\right)=: H_{0}$ is in diagonal form, or in its rest frame. We will denote by $E_{a}=\left(E_{1}, E_{2}, E_{3}\right)$ the eigenvalues.

If $|n\rangle$ denotes a normalized vector in the Hilbert space $\mathcal{H}$ on which $H$ operates, a natural connection on $\mathcal{H}$ associated with the projection $\pi: \mathcal{H} \backslash\{0\} \rightarrow \mathcal{P}(\mathcal{H}$ ) (or for the principal bundle $\mathbf{C}^{*} \rightarrow \mathcal{H} \backslash\{0\} \rightarrow \mathcal{P}(\mathcal{H})$ ) is defined by the parallel transport condition:

$$
\langle n \mid \dot{n}\rangle=0 \Leftrightarrow\langle n \mid d n\rangle=0,
$$

where $\dot{n}$ is evaluated along the "transporting path." If the transport is unitary we may identify $|n\rangle$ with $|a ; \xi\rangle$ with $\xi=\xi(t)$. We may also consider (cf. Sec. 3) the "instantaneous" matrix $A(\xi)$ such that

$$
|a ; \xi\rangle=A(\xi)|a\rangle,
$$

where $\{|a\rangle\}$ is the standard basis (50). As discussed in Sec. 3, $A(\xi)$ brings by conjugation $H(\xi)$ to its diagonal form

$$
H(\xi)=A(\xi) H_{0} A(\xi)^{\dagger}
$$


and is determined only up to right multiplication by the isotropy group of $H_{0}$. Notice that this equation leads to

$$
d H=\left[d A A^{\dagger}, H\right]=A\left[A^{\dagger} d A, H_{0}\right] A^{\dagger} .
$$

It is clear now that the parallel transport condition leads to $\langle a ; \xi|(d / d t)| a ; \xi\rangle=0$ and, equivalently, to

$$
\langle a ; \xi|d A| a\rangle=0
$$

or to

$$
\left\langle a\left|A^{\dagger} d A\right| a\right\rangle=0 .
$$

Written in this way, this exhibits $A^{\dagger} d A$ as a left-invariant one-form, that can be written as: $A^{\dagger} d A=\theta^{r} \lambda_{r}$ in a basis $\left\{\theta^{r}\right\}$ of left-invariant one-forms. Taking the exterior differential and using again: $d A^{\dagger}=-A^{\dagger} d A A^{\dagger}$, we may write the differential of $A^{\dagger} d A$ as $d\left(A^{\dagger} d A\right)=-A^{\dagger} d A \wedge A^{\dagger} d A$. To make connection with, e.g. the discussion of Secs. 2 and 4 , let us remark that the symplectic structure on the orbit through $H_{0}$ (or $H$ for that matter) is given precisely by

$$
\omega_{H_{0}}=d \operatorname{Tr}\left\{H_{0} A^{\dagger} d A\right\}=-\operatorname{Tr}\left\{H_{0} A^{\dagger} d A \wedge A^{\dagger} d A\right\} .
$$

By using the eigenvalue equation for $H$ we find

$$
d H|a ; \xi\rangle=E_{a} d|a ; \xi\rangle-H d|a ; \xi\rangle
$$

whence, taking scalar products

$$
\langle b ; \xi|d H| a ; \xi\rangle=\left(E_{a}-E_{b}\right)\langle b ; \xi|d| a ; \xi\rangle .
$$

Therefore

$$
\frac{\left\langle b ; \xi\left|A\left[A^{\dagger} d A, H_{0}\right] A^{\dagger}\right| a ; \xi\right\rangle}{E_{a}-E_{b}}=\langle b ; \xi|d| a ; \xi\rangle=\frac{\left\langle b\left|\left[A^{\dagger} d A, H_{0}\right]\right| a\right\rangle}{E_{a}-E_{b}} .
$$

Going back now to the form that has been given initially for the geometric-phase (or curvature) two-forms $V^{(a)}$, we see that we can rewrite them as the imaginary parts of

$$
\widetilde{V}^{(a)}=\sum_{b \neq a} \frac{\left\langle a ; \xi\left|\left[d A A^{\dagger}, H\right]\right| b ; \xi\right\rangle \wedge\left\langle b ; \xi\left|\left[d A A^{\dagger}, H\right]\right| a ; \xi\right\rangle}{E_{a b^{2}}} .
$$

Using explicitly $\left[d A A^{\dagger}, H\right]=d A A^{\dagger} H-H d A A^{\dagger}$, we find next

$$
\widetilde{V}^{(a)}=\sum_{b \neq a}\left\langle a ; \xi\left|d A A^{\dagger}\right| b ; \xi\right\rangle \wedge\left\langle b ; \xi\left|d A A^{\dagger}\right| a ; \xi\right\rangle
$$

i.e.

$$
\widetilde{V}^{(a)}=\left\langle a ; \xi\left|d A A^{\dagger} \wedge d A A^{\dagger}\right| a ; \xi\right\rangle-\left\langle a ; \xi\left|d A A^{\dagger}\right| a ; \xi\right\rangle\left\langle a ; \xi\left|d A A^{\dagger}\right| a ; \xi\right\rangle
$$

and eventually

$$
\widetilde{V}^{(a)}=\operatorname{Tr}\left\{|a ; \xi\rangle\langle a ; \xi| d A A^{\dagger} \wedge d A A^{\dagger}\right\}=\operatorname{Tr}\left\{|a ; \xi\rangle\langle a ; \xi| A^{\dagger} d A \wedge A^{\dagger} d A\right\} .
$$


Thus,

$$
\sum_{a} E_{a} V^{(a)}=\operatorname{Im} \operatorname{Tr}\left\{H_{0} A^{\dagger} d A \wedge A^{\dagger} d A\right\}
$$

gives the required relationship between the symplectic structure on the coadjoint orbit through $H_{0}$ and the curvature forms of the Berry phase connection. In a sense, this is also the generalized "sum rule" among the three Berry phases that was mentioned at the beginning of this section.

We can now relate the expressions of the coefficients of $V^{(a)}$ in a general frame and in their rest frame by use of Eqs. (51) and (A.8):

$$
\begin{aligned}
V_{r s}^{(a)}(\boldsymbol{\xi}) & =D_{r u}(A(\boldsymbol{\xi})) D_{s v}(A(\boldsymbol{\xi})) V_{u v}^{(a)}\left(\boldsymbol{\xi}^{(0)}\right), \\
V_{r s}^{(a)}\left(\boldsymbol{\xi}^{(0)}\right) & =\frac{1}{4} \operatorname{Im} \sum_{b \neq a} \frac{\left(\lambda_{u}\right)_{a b}\left(\lambda_{v}\right)_{b a}-\left(\lambda_{v}\right)_{a b}\left(\lambda_{u}\right)_{b a}}{E_{a b}\left(\boldsymbol{\xi}^{(0)}\right)^{2}} .
\end{aligned}
$$

The ambiguity of $A(\boldsymbol{\xi})$ up to an $\mathrm{U}(1) \times \mathrm{U}(1)$ element on the right does not affect this relation, since this is also the stability group of $\boldsymbol{\xi}^{(0)}$. By a double application of (96), for any $A \in \mathrm{SU}(3)$, we can relate $V_{r s}^{(a)}(D(A) \boldsymbol{\xi})$ to $V_{u v}^{(a)}(\boldsymbol{\xi})$. The argument rests on the fact that, if $\boldsymbol{\xi}^{\prime}=D(A) \boldsymbol{\xi}$, then $A\left(\boldsymbol{\xi}^{\prime}\right)$ which connects $\boldsymbol{\xi}^{\prime}$ to $\boldsymbol{\xi}^{(0)}$, can differ from $A(\boldsymbol{\xi})$ only by an element of $\mathrm{U}(1) \times \mathrm{U}(1)$ on the right:

$$
A(D(A) \boldsymbol{\xi})=A(\boldsymbol{\xi}) L, \quad L \in \mathrm{U}(1) \times \mathrm{U}(1) .
$$

Thus $L$ is a "Wigner rotation." Using $D(L) \boldsymbol{\xi}^{(0)}=\boldsymbol{\xi}^{(0)}$, we easily find

$$
\boldsymbol{\xi}^{\prime}=D(A) \boldsymbol{\xi}: V_{r s}^{(a)}\left(\boldsymbol{\xi}^{\prime}\right)=D_{r u}(A) D_{s v}(A) V_{u v}^{(a)}(\boldsymbol{\xi}) .
$$

Thus $V_{r s}^{(a)}(\boldsymbol{\xi})$ explicitly transforms in a covariant manner and at $\boldsymbol{\xi}^{(0)}$ it is $\mathrm{U}(1) \times \mathrm{U}(1)$ invariant.

The $\mathrm{U}(1) \times \mathrm{U}(1)$ subgroup in $\mathrm{SU}(3)$ is generated by $\lambda_{3}$ and $\lambda_{8}$. On a general $\boldsymbol{\xi}$ the effects are

$$
\begin{aligned}
D\left(e^{i \alpha \lambda_{3}}\right) \boldsymbol{\xi}= & \left(\xi_{1} \cos (2 \alpha)+\xi_{2} \sin (2 \alpha), \xi_{2} \cos (2 \alpha)\right. \\
& -\xi_{1} \sin (2 \alpha), \xi_{3}, \xi_{4} \cos \alpha+\xi_{5} \sin \alpha, \xi_{5} \cos \alpha \\
& \left.-\xi_{4} \sin \alpha, \xi_{6} \cos \alpha-\xi_{7} \sin \alpha, \xi_{7} \cos \alpha+\xi_{6} \sin \alpha, \xi_{8}\right) \\
D\left(e^{i \beta \lambda_{8}}\right) \boldsymbol{\xi}= & \left(\xi_{1}, \xi_{2}, \xi_{3}, \xi_{4} \cos (\sqrt{3} \beta)+\xi_{5} \sin (\sqrt{3} \beta), \xi_{5} \cos (\sqrt{3} \beta)\right. \\
& -\xi_{4} \sin (\sqrt{3} \beta), \xi_{6} \cos (\sqrt{3} \beta) \\
& \left.+\xi_{7} \sin (\sqrt{3} \beta), \xi_{7} \cos (\sqrt{3} \beta)-\xi_{6} \sin (\sqrt{3} \beta), \xi_{8}\right)
\end{aligned}
$$

Invariance under these transformations and antisymmetry in $r, s$ imply that the only nonzero components of $V_{r s}^{(a)}\left(\boldsymbol{\xi}^{(0)}\right)$ are possibly those with $r s=12,21,45,54$, 
$67,76,38,83$. Detailed calculations using (96) lead to the following independent nonvanishing elements:

$$
\begin{aligned}
& V_{12}^{(1)}\left(\boldsymbol{\xi}^{(0)}\right)=\frac{1}{2 E_{12}^{2}}, \quad V_{45}^{(1)}\left(\boldsymbol{\xi}^{(0)}\right)=\frac{1}{2 E_{13}^{2}}, \quad V_{67}^{(1)}\left(\boldsymbol{\xi}^{(0)}\right)=0, \\
& V_{12}^{(2)}\left(\boldsymbol{\xi}^{(0)}\right)=-\frac{1}{2 E_{12}^{2}}, \quad V_{45}^{(2)}\left(\boldsymbol{\xi}^{(0)}\right)=0, \quad V_{67}^{(2)}\left(\boldsymbol{\xi}^{(0)}\right)=\frac{1}{2 E_{23}^{2}}, \\
& V_{12}^{(3)}\left(\boldsymbol{\xi}^{(0)}\right)=0, \quad V_{45}^{(3)}\left(\boldsymbol{\xi}^{(0)}\right)=-\frac{1}{2 E_{13}^{2}}, \quad V_{67}^{(3)}\left(\boldsymbol{\xi}^{(0)}\right)=-\frac{1}{2 E_{23}^{2}} .
\end{aligned}
$$

We have now to use (95) to transform back from the rest frame to the general frame, knowing that we are dealing with invariant quantities obeying Eq. (98). This involves some SU(3) irreducible tensor analysis which we develop in the next section.

\section{SU(3) Tensor Analysis of the Geometric Phase Two-Forms}

The transformation law (98) shows that, for each level $a, V_{r s}^{(a)}(\boldsymbol{\xi})$ is a second rank tensor over the octet representation of SU(3). In App. A, we describe how such a tensor can be decomposed into $\mathrm{SU}(3)$-irreducible components belonging to the decouplet $(\underline{10})$, antidecouplet $\left(\underline{10}^{\star}\right)$ and octet $(\underline{8})$ UIR's of $\mathrm{SU}(3)$, and then can be recovered by combinations of these components. Based on the matrix elements $V_{r s}^{(a)}\left(\boldsymbol{\xi}^{(0)}\right)$ given in (101), we must compute the nonvanishing irreducible tensor components in the rest frame, for each $a$, and then use Eq. (96) to get the expression for general $\boldsymbol{\xi}$.

It is convenient to interchangeably use octet indices $r, s=1, \ldots, 8$ and contravariant and covariant $\mathrm{SU}(3)$ tensor indices $b, c, d, e, f=1,2,3$. We find that, in the rest frame and for each level $a=1,2,3$, the only nonvanishing components of the $(\underline{10})$ and $\left(\underline{10}^{\star}\right)$ tensors $W^{(a) b c d}, \bar{W}_{b c d}^{(a)}$ are the 123 components; these are the only $\mathrm{U}(1) \times \mathrm{U}(1)$ invariant ones. As for the octet parts, in all cases $X_{r}^{(a)}$ turns out to be diagonal (and traceless), so in the octet notation this means that only the $r=3$ and $r=8$ components remain. Again by (99) and (100) these are $\mathrm{U}(1) \times \mathrm{U}(1)$ invariant.

We list in formula (102) the nonvanishing rest frame irreducible tensor components for each $V_{r s}^{(a)}\left(\boldsymbol{\xi}^{(0)}\right)$

$$
\begin{array}{ccc}
W^{(a) 123} & \bar{W}_{123}^{(a)} & X_{8}^{(a)} \\
V_{r s}^{(1)}\left(\boldsymbol{\xi}^{(0)}\right) i\left(\frac{1}{E_{13}^{2}}-\frac{1}{E_{12}^{2}}\right) i\left(\frac{1}{E_{12}^{2}}-\frac{1}{E_{13}^{2}}\right)-\frac{1}{E_{12}^{2}}-\frac{1}{2 E_{13}^{2}} & -\frac{\sqrt{3}}{2} \frac{1}{E_{13}^{2}} \\
V_{r s}^{(2)}\left(\boldsymbol{\xi}^{(0)}\right) i\left(\frac{1}{E_{12}^{2}}-\frac{1}{E_{23}^{2}}\right) i\left(\frac{1}{E_{23}^{2}}-\frac{1}{E_{12}^{2}}\right) \frac{1}{E_{12}^{2}}+\frac{1}{2 E_{23}^{2}} & -\frac{\sqrt{3}}{2} \frac{1}{E_{23}^{2}} \\
V_{r s}^{(3)}\left(\boldsymbol{\xi}^{(0)}\right) i\left(\frac{1}{E_{23}^{2}}-\frac{1}{E_{13}^{2}}\right) i\left(\frac{1}{E_{13}^{2}}-\frac{1}{E_{23}^{2}}\right) \frac{1}{2 E_{13}^{2}}-\frac{1}{2 E_{23}^{2}} \frac{\sqrt{3}}{2}\left(\frac{1}{E_{13}^{2}}+\frac{1}{E_{23}^{2}}\right) .
\end{array}
$$

We note that the $(\underline{10})$ and $\left(\underline{10}^{\star}\right)$ components are purely imaginary. 
Now we have to obtain $V_{r s}^{(a)}(\boldsymbol{\xi})$ in a general frame, expressing them as far as possible explicitly in terms of $\boldsymbol{\xi}$. First we consider the contributions from the octet components $X_{r}^{(a)}\left(\boldsymbol{\xi}^{(0)}\right)$. To begin with, for each $a$ decompose $X_{r}^{(a)}\left(\boldsymbol{\xi}^{(0)}\right)$ as a linear combination of the available rest frame octet vectors $\boldsymbol{\xi}^{(0)}$ and $\boldsymbol{\eta}^{(0)}$, where $\boldsymbol{\eta}^{(0)}=$ $\boldsymbol{\xi}^{(0)} * \boldsymbol{\xi}^{(0)}$. After some algebra we find

$$
\begin{aligned}
X_{r}^{(a)}\left(\boldsymbol{\xi}^{(0)}\right) & =\left[\xi_{3}^{(0)}\left(\xi_{3}^{(0) 2}-3 \xi_{8}^{(0) 2}\right)\right]^{-1}\left(\lambda^{(a)} \xi_{r}^{(0)}+\mu^{(a)} \eta_{r}^{(0)}\right) \\
\lambda^{(1)} & =\frac{\sqrt{3} \eta_{3}^{(0)}-\eta_{8}^{(0)}}{2 E_{13}^{2}}-\frac{\eta_{8}^{(0)}}{E_{12}^{2}} \\
\lambda^{(2)} & =\frac{\sqrt{3} \eta_{3}^{(0)}+\eta_{8}^{(0)}}{2 E_{23}^{2}}+\frac{\eta_{8}^{(0)}}{E_{12}^{2}} \\
\lambda^{(3)} & =\frac{\eta_{8}^{(0)}-\sqrt{3} \eta_{3}^{(0)}}{2 E_{13}^{2}}-\frac{\eta_{8}^{(0)}+\sqrt{3} \eta_{3}^{(0)}}{2 E_{23}^{2}} \\
\mu^{(1)} & =\frac{\xi_{8}^{(0)}}{E_{12}^{2}}+\frac{\xi_{8}^{(0)}-\sqrt{3} \xi_{3}^{(0)}}{2 E_{13}^{2}} \\
\mu^{(2)} & =-\frac{\xi_{8}^{(0)}}{E_{12}^{2}}-\frac{\xi_{8}^{(0)}+\sqrt{3} \xi_{3}^{(0)}}{2 E_{23}^{2}} \\
\mu^{(3)} & =\frac{\sqrt{3} \xi_{3}^{(0)}-\xi_{8}^{(0)}}{2 E_{13}^{2}}+\frac{\sqrt{3} \xi_{3}^{(0)}+\xi_{8}^{(0)}}{2 E_{23}^{2}}
\end{aligned}
$$

Apart from the expected squares of energy denominators, combinations of the components of $\boldsymbol{\xi}^{(0)}$ and $\boldsymbol{\eta}^{(0)}$ appear. They are expressible in terms of $E_{a b}$ using

$$
\begin{array}{cl}
\xi_{3}^{(0)}=E_{12}, & \xi_{8}^{(0)}=\frac{E_{13}+E_{23}}{2 \sqrt{3}}, \\
\eta_{3}^{(0)}=\frac{E_{12}\left(E_{13}+E_{23}\right)}{\sqrt{3}}, \quad \eta_{8}^{(0)}=E_{12}^{2}-\frac{\left(E_{13}+E_{23}\right)^{2}}{12}, \\
\xi_{3}^{(0)}\left(\xi_{3}^{(0) 2}-3 \xi_{8}^{(0) 2}\right)=-4 E_{12} E_{13} E_{23} .
\end{array}
$$

Combining the appropriate parts of Eqs. (B.3), (B.4) and (B.6), we see that in a general frame the octet part of $V_{r s}^{(a)}(\boldsymbol{\xi})$ is given by

$$
-\frac{1}{3} f_{r s t} X_{t}^{(a)}(\boldsymbol{\xi})=\frac{1}{12 E_{12} E_{13} E_{23}} f_{r s t}\left(\lambda^{(a)} \xi_{t}+\mu^{(a)} \eta_{t}\right) \text {. }
$$

Actually the combinations of $\boldsymbol{\xi}$ and $\boldsymbol{\eta}=\boldsymbol{\xi} * \boldsymbol{\xi}$ occurring here become singular near the regions of double degeneracy, $E_{12} \rightarrow 0$ or $E_{23} \rightarrow 0$. We examine these details in Sec. 7. 
The $(\underline{10})$ and $\left(\underline{10}^{\star}\right)$ contributions to $V_{r s}^{(a)}(\boldsymbol{\xi})$ are more subtle in structure. We have seen that in the rest frame the only nonzero components of $W^{(a) b c d}$ and $\bar{W}_{b c d}^{(a)}$ are the ones with $b c d=123$ (or permutations thereof). Let us define a numerical tensor of type $(\underline{10})$ in the rest frame by

$$
\delta^{a b c}= \begin{cases}1 & \text { if } a b c=\text { any permutation of } 123 \\ 0 & \text { otherwise }\end{cases}
$$

Thus the only independent nonzero component of $\delta^{a b c}$ is the one invariant under $\mathrm{U}(1) \times \mathrm{U}(1)$, the stability group of $\boldsymbol{\xi}^{(0)}$. Then in the general frame we define the (10) tensor

$$
\Delta^{a b c}(\boldsymbol{\xi})=A_{d}^{a}(\boldsymbol{\xi}) A_{e}^{b}(\boldsymbol{\xi}) A_{f}^{c}(\boldsymbol{\xi}) \delta^{d e f} .
$$

The ambiguity in $A(\boldsymbol{\xi})$ up to a $\mathrm{U}(1) \times \mathrm{U}(1)$ element on the right leaves $\delta^{\text {def }}$ unaffected. Therefore it is consistent to maintain that the quantities $\Delta^{a b c}(\boldsymbol{\xi})$ are the components of a decouplet or ( $\underline{10})$ tensor under $\boldsymbol{\xi} \rightarrow \boldsymbol{\xi}^{\prime}=A \boldsymbol{\xi}$ and, as written, are well-defined functions of the octet vector $\boldsymbol{\xi}$, in spite of the practical difficulty in developing the expression (107) further. In a similar way, we set up a rest frame numerical $\left(\underline{10}^{\star}\right)$ tensor $\bar{\delta}_{a b c}$, and then transport it to a general frame to get a $\left(\underline{10}^{\star}\right)$ tensor $\bar{\Delta}_{a b c}(\boldsymbol{\xi})$ :

$$
\begin{aligned}
\bar{\delta}_{a b c} & = \begin{cases}1 & \text { if } a b c=\text { any permutation of } 123, \\
0 & \text { otherwise }\end{cases} \\
\bar{\Delta}_{a b c}(\boldsymbol{\xi}) & =A_{a}^{d}(\boldsymbol{\xi}) A_{b}^{e}(\boldsymbol{\xi}) A_{c}^{f}(\boldsymbol{\xi}) \delta_{d e f} .
\end{aligned}
$$

Picking up the terms in Eq. (B.6) involving $W$ and $\bar{W}$, and using (102), we get the remaining decouplet parts of $V_{d e}^{(a) b c}(\boldsymbol{\xi})$, expressed in tensor index notation:

$$
\frac{i v^{(a)}}{6}\left(\epsilon_{d e f} \Delta^{b c f}(\boldsymbol{\xi})-\epsilon^{b c f} \bar{\Delta}_{d e f}(\boldsymbol{\xi})\right)
$$

with

$$
v^{(1)}=\frac{1}{E_{13}^{2}}-\frac{1}{E_{12}^{2}}, \quad v^{(2)}=\frac{1}{E_{12}^{2}}-\frac{1}{E_{23}^{2}}, \quad v^{(3)}=\frac{1}{E_{23}^{2}}-\frac{1}{E_{13}^{2}} .
$$

The complete two-forms $V_{r s}^{(a)}(\boldsymbol{\xi})$ are obtained by putting together the expressions in Eqs. (105) and (110).

\section{Behavior Near Double Degeneracy}

From the description of the eigenvalue spectrum of $H(\boldsymbol{\xi})$ in Sec. 3, it is clear that the energy differences obey

$$
E_{12}, \quad E_{23} \geq 0, \quad E_{13}=E_{12}+E_{23} \geq E_{12}, \quad E_{23} .
$$

The vanishing of $E_{13}$ occurs only at the point of triple degeneracy $\boldsymbol{\xi}=0$, the origin in $\mathbf{R}^{8}$. Away from this point, $E_{13}$ is always strictly positive. However, as pointed 
out in Sec. 3, there are particular directions in $\mathbf{R}^{8}$ along which either $E_{12}$ or $E_{23}$ vanishes, signalling a double degeneracy. These are the two separate four-parameter sets of directions comprising the regions $\Sigma_{12}, \Sigma_{23}$ defined in Eqs. (47) and (48). As explained in App. A, these directions map out the two four-dimensional regions in $S^{7}$ which correspond to the two singular orbits in the Lie algebra su(3) of $\mathrm{SU}(3)$, each realizing the coset space $\mathrm{SU}(3) / \mathrm{U}(2)$. We can exhibit the structures of the two basic energy differences $E_{12}, E_{23}$ in the vicinity of these regions, expressing them in terms of the two $\mathrm{SU}(3)$ invariants $\boldsymbol{\xi}^{2}$ and $\boldsymbol{\xi} \cdot \boldsymbol{\xi} * \boldsymbol{\xi}$. Starting with Eqs. (38) and (42) and expanding them near $\phi=\pi / 6$ and $\phi=\pi / 2$ respectively, we find

$$
\begin{aligned}
& \phi \approx \frac{\pi}{6}, \quad \boldsymbol{\xi} \text { near } \Sigma_{12}: E_{12}(\boldsymbol{\xi}) \approx \frac{\sqrt{2}}{3} \frac{\left(\xi^{3}+\boldsymbol{\xi} \cdot \boldsymbol{\xi} * \boldsymbol{\xi}\right)^{1 / 2}}{\xi^{1 / 2}} \\
& \phi \approx \frac{\pi}{2}, \quad \boldsymbol{\xi} \text { near } \Sigma_{23}: E_{23}(\boldsymbol{\xi}) \approx \frac{\sqrt{2}}{3} \frac{\left(\xi^{3}-\boldsymbol{\xi} \cdot \boldsymbol{\xi} * \boldsymbol{\xi}\right)^{1 / 2}}{\xi^{1 / 2}} .
\end{aligned}
$$

Now we analyze in more detail the situation near, say, the upper double degeneracy, when $\boldsymbol{\xi}$ lies close to $\Sigma_{12}$. First we deal with the octet or $X^{(a)}$ contribution to $V_{r s}^{(a)}(\boldsymbol{\xi})$ in this limit, and later look at the $(\underline{10})$ and $\left(\underline{10}^{\star}\right)$ contributions. The aim is to trace how the two-level monopole structure is recovered from the present three-level formalism. It is adequate to work in the rest frame $\boldsymbol{\xi}=\boldsymbol{\xi}^{(0)}$ and use Eq. (103), since the transition to a general frame via $\mathrm{SU}(3)$ cannot introduce any singular factors. Let us in the following denote the small energy difference $E_{12}$ by $\epsilon$. The prefactor in Eq. (103) introduces an explicit $1 / \epsilon$ factor in the expression for $X^{(a)}$ :

$$
\left[\xi_{3}^{(0)}\left(\xi_{3}^{(0) 2}-3 \xi_{8}^{(0) 2}\right)\right]^{-1}=-\frac{1}{4 \epsilon E_{13} E_{23}} .
$$

This must be balanced by matching powers of $\epsilon$ from the remaining parts of Eq. (103), since we know from the general formula (77) that nothing more singular than $1 / \epsilon^{2}$ can appear in the two-form $V^{(a)}$. This is indeed borne out by detailed calculations. We find, remembering that $E_{12}=\epsilon$ is the small parameter, that the $3 \times 3$ Hermitian matrices $X^{(a)}\left(\xi^{(0)}\right)$ have the following structures:

$$
\begin{aligned}
X^{(1)}\left(\xi^{(0)}\right) & =-\frac{1}{4 E_{13} E_{23}}\left[2\left(2 \frac{E_{13}^{2}}{\epsilon^{2}}-2 \frac{E_{13}}{\epsilon}+1-\frac{\epsilon}{E_{13}}\right) \lambda_{3}+2 \sqrt{3}\left(1-\frac{\epsilon}{E_{13}}\right) \lambda_{8}\right] \\
& =-\frac{\lambda_{3}}{\epsilon^{2}}+\text { nonsingular terms } \\
X^{(2)}\left(\xi^{(0)}\right) & =-\frac{1}{4 E_{13} E_{23}}\left[-2\left(2 \frac{E_{23}^{2}}{\epsilon^{2}}+2 \frac{E_{23}}{\epsilon}+1+\frac{\epsilon}{E_{23}}\right) \lambda_{3}+2 \sqrt{3}\left(1+\frac{\epsilon}{E_{23}}\right) \lambda_{8}\right] \\
& =\frac{\lambda_{3}}{\epsilon^{2}}+\text { nonsingular terms } \\
X^{(3)}\left(\xi^{(0)}\right) & =\text { only nonsingular terms. }
\end{aligned}
$$


Therefore from Eq. (105) the leading nonvanishing octet contributions to $V_{r s}^{(a)}\left(\boldsymbol{\xi}^{(0)}\right)$, apart from antisymmetry in $r$ and $s$, are then:

$$
\begin{aligned}
& V_{12}^{(1)}\left(\boldsymbol{\xi}^{(0)}\right)=\frac{1}{3 \epsilon^{2}}, \quad V_{45}^{(1)}\left(\boldsymbol{\xi}^{(0)}\right)=\frac{1}{6 \epsilon^{2}}, \quad V_{67}^{(1)}\left(\boldsymbol{\xi}^{(0)}\right)=-\frac{1}{6 \epsilon^{2}}, \\
& V_{12}^{(2)}\left(\boldsymbol{\xi}^{(0)}\right)=-\frac{1}{3 \epsilon^{2}}, \quad V_{45}^{(2)}\left(\boldsymbol{\xi}^{(0)}\right)=-\frac{1}{6 \epsilon^{2}}, \quad V_{67}^{(2)}\left(\boldsymbol{\xi}^{(0)}\right)=\frac{1}{6 \epsilon^{2}}, \\
& V_{r s}^{(3)}\left(\boldsymbol{\xi}^{(0)}\right)=\text { nonsingular . }
\end{aligned}
$$

On the other hand, from (102) and (111) we find that the leading nonvanishing $(\underline{10})$ and $\left(\underline{10}^{\star}\right)$ contributions to $V_{r s}^{(a)}\left(\boldsymbol{\xi}^{(0)}\right)$, again apart from antisymmetry in $r$ and $s$, are as follows:

$$
\begin{array}{rlrl}
V_{12}^{(1)}\left(\boldsymbol{\xi}^{(0)}\right) & =\frac{1}{6 \epsilon^{2}}, & V_{45}^{(1)}\left(\boldsymbol{\xi}^{(0)}\right)=-\frac{1}{6 \epsilon^{2}}, & V_{67}^{(1)}\left(\boldsymbol{\xi}^{(0)}\right)=\frac{1}{6 \epsilon^{2}} \\
V_{12}^{(2)}\left(\boldsymbol{\xi}^{(0)}\right) & =-\frac{1}{6 \epsilon^{2}}, & V_{45}^{(2)}\left(\boldsymbol{\xi}^{(0)}\right)=\frac{1}{6 \epsilon^{2}}, & V_{67}^{(2)}\left(\boldsymbol{\xi}^{(0)}\right)=-\frac{1}{6 \epsilon^{2}} \\
V_{r s}^{(3)}\left(\boldsymbol{\xi}^{(0)}\right) & =\text { nonsingular. }
\end{array}
$$

Adding the two sets of contributions we see that for $\boldsymbol{\xi}$ near $\Sigma_{12}$, in the rest frame, the surviving singular terms in the two-forms $V_{r s}^{(a)}\left(\boldsymbol{\xi}^{(0)}\right)$ are very few

$$
V_{12}^{(1)}\left(\boldsymbol{\xi}^{(0)}\right)=-V_{12}^{(2)}\left(\boldsymbol{\xi}^{(0)}\right)=\frac{1}{2 \epsilon^{2}} .
$$

We can see how the different irreducible SU(3) tensor components combine in just the right manner to reproduce the three-dimensional magnetic monopole type of singularity in the two-forms $V_{r s}^{(a)}\left(\boldsymbol{\xi}^{(0)}\right)$ when we are near the point of doubledegeneracy. Limiting ourself to $\mathrm{SU}(2)$ transformations in the 12 subspace in $\mathrm{SU}(3)$, it is evident that the terms $V_{12}^{(1,2)}\left(\boldsymbol{\xi}^{(0)}\right)$ of Eq. (121) give rise to the familiar threedimensional monopole field, expressed however in eight-dimensional space using the relations in (76). Thus they are singular not only at a single point but all over the region $\Sigma_{12} \subset \mathbf{R}^{8}-\{\mathbf{0}\}$.

\section{Concluding Remarks}

We have presented a study of two aspects of multilevel quantum systems, unified by the geometrical features of coadjoint orbits in the Lie algebra of the unitary groups $U(n), \mathrm{SU}(n)$. These are the properties of unitary von Neumann evolution of general pure or mixed states for such systems; and in the $n=3$ case the detailed structures of the geometric phases in the neighborhood of degeneracies.

We have examined the structures of the two-forms or covariant antisymmetric tensor fields in parameter space, whose fluxes through any closed circuit give the corresponding quantum adiabatic geometric phases when one is in the vicinity of a point of three-level degeneracy. In comparison with the three-dimensional monopole singularity in the two-level case, here one has a much richer structure with many 
new features. To begin with, the parameter space is eight-dimensional. In the spirit of the von Neumann-Wigner theorem, double degeneracies occur along two fourparameter sets of singular rays, $\Sigma_{12}$ and $\Sigma_{23}$, in parameter space; while the triple degeneracy occurs just at a single point $\boldsymbol{\xi}=\mathbf{0}$. Beyond this there is a rich $\mathrm{SU}(3)$ tensor structure in the relevant two-forms. Whereas for the two-level case the result was a simple radial "vector" field, here we have three independent irreducible tensor contributions belonging to the tensor types $(\underline{8}),(\underline{10})$ and $\left(\underline{10}^{\star}\right)$. Overall reality makes the last two complex conjugates of one another, but intrinsically they should be considered as independent of one another and of the (모) contribution.

The complexity of the expressions we have obtained is unavoidable and automatically belongs to the next most interesting case from the point of view of general quantum mechanics after the two-level degeneracy. We have shown that they contain within them, embedded in intricate ways, the monopole of the double degeneracy problem when one is near one of the regions $\Sigma_{12}$ and $\Sigma_{23}$, in parameter space. However we emphasize that in the complete eight-dimensional picture these are far from being point singularities. We can explain the complexity of the present expressions by saying that they have to contain the earlier monopole results in certain limiting situations, and then go beyond them to handle a triple degeneracy.

\section{Appendix A}

We collect here some basic information about the defining and adjoint representations of $\mathrm{SU}(3)$. The group $\mathrm{SU}(3)$ is defined as follows:

$$
\mathrm{SU}(3)=\left\{A=3 \times 3 \text { complex matrix: } A^{\dagger} A=\mathbf{I}, \operatorname{det} A=1\right\} .
$$

The eight independent Hermitian traceless generators are the Gell-Mann $\lambda$ matrices:

$$
\begin{array}{rlrl}
\lambda_{1} & =\left[\begin{array}{rrr}
0 & 1 & 0 \\
1 & 0 & 0 \\
0 & 0 & 0
\end{array}\right], & \lambda_{2}=\left[\begin{array}{rrr}
0 & -i & 0 \\
i & 0 & 0 \\
0 & 0 & 0
\end{array}\right], \\
\lambda_{3}=\left[\begin{array}{rrr}
1 & 0 & 0 \\
0 & -1 & 0 \\
0 & 0 & 0
\end{array}\right], & \lambda_{4}=\left[\begin{array}{lll}
0 & 0 & 1 \\
0 & 0 & 0 \\
1 & 0 & 0
\end{array}\right], \\
\lambda_{5}=\left[\begin{array}{rrr}
0 & 0 & -i \\
0 & 0 & 0 \\
i & 0 & 0
\end{array}\right], & \lambda_{6}=\left[\begin{array}{rrr}
0 & 0 & 0 \\
0 & 0 & 1 \\
0 & 1 & 0
\end{array}\right], \\
\lambda_{7}=\left[\begin{array}{rrr}
0 & 0 & 0 \\
0 & 0 & -i \\
0 & i & 0
\end{array}\right], & \lambda_{8}=\frac{1}{\sqrt{3}}\left[\begin{array}{rrr}
1 & 0 & 0 \\
0 & 1 & 0 \\
0 & 0 & -2
\end{array}\right]
\end{array}
$$

that obey the following rule:

$$
\operatorname{Tr}\left\{\lambda_{r} \lambda_{s}\right\}=2 \delta_{r s}
$$


Their commutation and anticommutation relations involve the completely antisymmetric structure constants $f_{r s t}$ and the completely symmetric $d$-symbols $d_{r s t}$ :

$$
\left[\lambda_{r}, \lambda_{s}\right]=2 i f_{r s t} \lambda_{t}
$$

and

$$
\left\{\lambda_{r}, \lambda_{s}\right\}=\frac{4}{3} \delta_{r s}+2 d_{r s t} \lambda_{t}
$$

$(r, s, t=1, \ldots, 8)$ where the numerical values of the independent $f_{r s t}$ and $d_{r s t}$ are

$$
\begin{gathered}
f_{123}=1, \quad f_{458}=f_{678}=\frac{\sqrt{3}}{2}, \\
f_{147}=f_{246}=f_{257}=f_{345}=f_{516}=f_{637}=\frac{1}{2}
\end{gathered}
$$

and

$$
\begin{aligned}
d_{118} & =d_{228}=d_{338}=-d_{888}=\frac{1}{\sqrt{3}}, \\
d_{448} & =d_{558}=d_{668}=d_{778}=-\frac{1}{2 \sqrt{3}}, \\
d_{146} & =d_{157}=-d_{247}=d_{256}=d_{344} \\
& =d_{355}=-d_{366}=-d_{377}=\frac{1}{2} .
\end{aligned}
$$

Under conjugation by any $A \in \mathrm{SU}(3)$ the $\lambda$ 's go into real orthogonal linear combinations of themselves

$$
\begin{aligned}
A^{\dagger} \lambda_{r} A & =D_{r s}(A) \lambda_{s}, \\
D_{r s}(A) & =\frac{1}{2} \operatorname{Tr}\left\{\lambda_{r} A \lambda_{s} A^{\dagger}\right\} \\
D(A)^{T} D(A) & =\mathbf{I} \\
D\left(A^{\prime}\right) D(A) & =D\left(A^{\prime} A\right)
\end{aligned}
$$

These matrices constitute the octet or adjoint representation of $\mathrm{SU}(3)$, actually a faithful representation of the quotient $\mathrm{SU}(3) / \mathbb{Z}_{3}$. They describe the action of $\mathrm{SU}(3)$ on a general eight component real octet vector, as a small subgroup of the full twenty eight parameter group $\mathrm{SO}(8)$. Given $\boldsymbol{\xi}^{1}, \boldsymbol{\xi}^{2} \in \mathbf{R}^{8}$, we can form the $\mathrm{SU}(3)$ (and $\mathrm{SO}(8)$ ) invariant inner product

$$
\boldsymbol{\xi}^{1} \cdot \boldsymbol{\xi}^{2}=\boldsymbol{\xi}_{r}^{1} \boldsymbol{\xi}_{r}^{2}
$$

the antisymmetric octet vector $\boldsymbol{\xi}^{1} \wedge \boldsymbol{\xi}^{2}$ using the structure constants

$$
\left(\boldsymbol{\xi}^{1} \wedge \boldsymbol{\xi}^{2}\right)_{r}=-\frac{1}{2} f_{r s t} \xi_{s}^{1} \xi_{t}^{2},
$$


as well as a symmetric octet vector $\boldsymbol{\xi}^{1} * \boldsymbol{\xi}^{2}$ using the $d$-symbols:

$$
\left(\boldsymbol{\xi}^{1} * \boldsymbol{\xi}^{2}\right)_{r}=\sqrt{3} d_{r s t} \xi_{s}^{1} \xi_{t}^{2} .
$$

The latter two definitions make sense only with respect to $\mathrm{SU}(3)$ and not $\mathrm{SO}(8)$.

From a given $\boldsymbol{\xi} \in \mathbf{R}^{8}$ one can construct the $\mathrm{SU}(3)$ invariant $|\xi|^{2}=\boldsymbol{\xi} \cdot \boldsymbol{\xi}$ and $\boldsymbol{\xi} \cdot \boldsymbol{\xi} * \boldsymbol{\xi}$. The action of $\mathrm{SU}(3)$ on $S^{7}$ is intricate. Denote by $\mathbf{n}$ a unit octet vector, so $|\mathbf{n}|^{2}=1$. The remaining $\mathrm{SU}(3)$ invariant is the cubic $\mathbf{n} \cdot \mathbf{n} * \mathbf{n}$ and it obeys

$$
-1 \leq \mathbf{n} \cdot \mathbf{n} * \mathbf{n} \leq 1 .
$$

As long as this invariant is in the open interval $(-1,1)$, the stability group of $\mathbf{n}$ is conjugate to $\mathrm{U}(1) \times \mathrm{U}(1) \subset \mathrm{SU}(3)$; hence the orbit of $\mathbf{n}$ under $\mathrm{SU}(3)$ action, $D(A) \mathbf{n} \forall A$, is six-dimensional and realizes the coset space $\mathrm{SU}(3) / \mathrm{U}(1) \times \mathrm{U}(1)$. The six dimensions of this orbit together with the variable parameter $\mathbf{n} \cdot \mathbf{n} * \mathbf{n}$ account for the seven dimensions of $S^{7}$ at generic points. At the end points of the interval in (A.12), the corresponding $\mathbf{n} \in S^{7}$ have four-dimensional stability groups, conjugate to $\mathrm{U}(2) \subset \mathrm{SU}(3)$. Correspondingly we have two very special and singular fourdimensional orbits of such vectors in $S^{7}$. In the notation of Eqs. (47) and (48), they are given by

$$
\begin{aligned}
& \Sigma_{12} \cap S^{7}=\left\{\mathbf{n} \in S^{7}: \mathbf{n} \cdot \mathbf{n} * \mathbf{n}=-1\right\}, \\
& \Sigma_{23} \cap S^{7}=\left\{\mathbf{n} \in S^{7}: \mathbf{n} \cdot \mathbf{n} * \mathbf{n}=+1\right\} .
\end{aligned}
$$

\section{Appendix B}

We develop here the algebraic tools to deal with the irreducible tensor components of a second rank antisymmetric $\mathrm{SU}(3)$ tensor over its octet representation.

For an octet vector we can pass between $X_{r}$ and its tensor components by using the $\lambda$ matrices:

$$
\begin{aligned}
X_{b}^{a} & =X_{r}\left(\lambda_{r}\right)_{a b}, \quad X_{a}^{a}=0, \\
X_{r} & =\frac{1}{2} \operatorname{Tr}\left\{X \lambda_{r}\right\}=\frac{1}{2} X_{b}^{a}\left(\lambda_{r}\right)_{a b}, \\
X_{r}^{\star} & =X_{r} \Leftrightarrow\left(X_{b}^{a}\right)^{\star}=X_{a}^{b} .
\end{aligned}
$$

For ease in writing, the matrix indices on the $\lambda$ 's are all given as subscripts.

Now let $T_{r s}=T_{r s}^{\star}=-T_{s r}$ be a real antisymmetric second rank tensor over the octet representation. Generalizing (B.1) we define its tensor components $T_{c d}^{a b}$ as follows:

$$
\begin{aligned}
& T_{c d}^{a b}=\left(T_{a b}^{c d}\right)^{\star}=-T_{d c}^{b a}=\left(\lambda_{r}\right)_{a c}\left(\lambda_{s}\right)_{b d} T_{r s}, \\
& T_{a c}^{a b}=T_{b a}^{a b}=0 \\
& T_{r s}=\frac{1}{4}\left(\lambda_{r}\right)_{c a}\left(\lambda_{s}\right)_{d b} T_{c d}^{a b} .
\end{aligned}
$$

Such a tensor has 28 independent real components. Now, the antisymmetric part of the direct product of two octet representations of $\mathrm{SU}(3)$ contains, upon reduction, the (complex) decouplet $\underline{10}$, the (conjugate) anti-decouplet $\underline{10}^{\star}$ and the (real) 
octet $\underline{8}$, once each. The counting agrees since reality of $T_{r s}$ implies that the $\underline{10}^{\star}$ components are complex conjugates of the $\underline{10}$ components. We project out the $\underline{10}$, $\underline{10}^{\star}$ and $\underline{8}$ components respectively, of $T_{r s}$ in the following way:

$$
\begin{aligned}
W^{a b c} & =\epsilon^{a d e} T_{d e}^{b c}+\epsilon^{b d e} T_{d e}^{c a}+\epsilon^{c d e} T_{d e}^{a b}, \\
\bar{W}_{a b c} & =W^{a b c^{*}}=\epsilon_{a d e} T_{b c}^{d e}+\epsilon_{b d e} T_{c a}^{d e}+\epsilon_{c d e} T_{a b}^{d e}, \\
X_{b}^{a} & =\left(X_{a}^{b}\right)^{\star}=i T_{c b}^{a c}, \quad X_{a}^{a}=0 .
\end{aligned}
$$

The tensor $W^{a b c}$ is fully symmetric in $a b c$, as is $\bar{W}_{a b c}$. The $\epsilon$ symbols are fully antisymmetric with $\epsilon^{123}=\epsilon_{123}=1$. Incidentally, the octet components $X_{r}$ of $X_{b}^{a}$ can be easily expressed in terms of $T_{r s}$ :

$$
\begin{aligned}
X_{r} & =\frac{i}{2}\left(\lambda_{r}\right)_{b a} T_{c b}^{a c}=\frac{i}{2}\left(\lambda_{r}\right)_{b a}\left(\lambda_{s}\right)_{a c}\left(\lambda_{t}\right)_{c b} T_{s t} \\
& =\frac{i}{2} \operatorname{Tr}\left\{\lambda_{r} \lambda_{s} \lambda_{t}\right\} T_{s t}=i\left(d_{r s t}+i f_{r s t}\right) V_{t}=-f_{r s t} V_{s t} .
\end{aligned}
$$

Now we reconstitute $T_{c d}^{a b}$ from $W, \bar{W}$ and $X$. As a first step we easily obtain

$$
\begin{gathered}
\epsilon_{c d e} W^{a b e}=3\left(T_{c d}^{a b}+T_{c d}^{b a}\right)+\delta_{c}^{a} T_{d e}^{e b}+\delta_{c}^{b} T_{d e}^{e a}-\delta_{d}^{a} T_{c e}^{e b}-\delta_{d}^{b} T_{c e}^{e a}, \\
\epsilon^{a b e} \bar{W}_{c b e}=3\left(T_{c d}^{a b}-T_{c d}^{b a}\right)-\delta_{c}^{a} T_{d e}^{e b}+\delta_{c}^{b} T_{d e}^{e a}-\delta_{d}^{a} T_{c e}^{e b}+\delta_{d}^{b} T_{c e}^{e a}
\end{gathered}
$$

The first expression is symmetric in $a b$ and antisymmetric in $c d$, while in the second it is the other way around. If we now add these two results and bring in the tensor $X_{b}^{a}$, we get the final formula:

$$
T_{c d}^{a b}=\frac{1}{6} \epsilon_{c d e} W^{a b e}+\frac{1}{6} \epsilon^{a b e} \bar{W}_{c d e}+\frac{i}{2}\left(\delta_{d}^{a} X_{c}^{b}-\delta_{c}^{b} X_{d}^{a}\right) .
$$

From here, via Eq. (B.2), we can obtain $T_{r s}$.

\section{Acknowledgments}

One of the authors (N. Mukunda) expresses grateful thanks to G. Marmo and G. Morandi for gracious hospitality at the Universities of Napoli and Bologna, where this work was initiated.

\section{References}

1. Y. Aharonov and J. Anandan, Phys. Rev. Lett. 58, 1593 (1987) (also reprinted in Ref. 25).

2. J. Anandan, J. Christian and K. Wanelik, Am. J. Phys. 65, 180 (1997).

3. Arvind, K. S. Mallesh and N. Mukunda, J. Phys. A30, 2417 (1997).

4. A. P. Balachandran, G. Marmo, B. S. Skagerstam and A. Stern, Gauge Symmetry and Fiber Bundles. Applications to Particle Dynamics (Springer-Verlag, Berlin, 1983).

5. A. P. Balachandran, G. Marmo, B. S. Skagerstam and A. Stern, Classical Topology and Quantum States (World Scientific, Singapore, 1991).

6. H. Bechmann-Pasquinucci and A. Peres, "Quantum criptography with 3-state systems," quant-ph/0001083 (2000). 
7. M. V. Berry, Proc. R. Soc. London A392, 45 (1984).

8. L. J. Boya, M. S. Byrd, M. Sims and E. C. G. Sudarshan, "Density matrices and geometric phases for $n$-state systems," quant-ph/9810084 (1998).

9. M. S. Byrd, J. Math. Phys. 39, 6125 (1998); 41, 1026 (2000).

10. M. S. Byrd and P. B. Slater, "Bures measures over the space of two and threedimensional density matrices," quant-ph/0004055 (2000).

11. M. S. Byrd and E. C. G. Sudarshan, J. Phys. A31, 9255 (1998).

12. I. L. Chuang and Y. Yamamoto, Phys. Rev. A52, 3489 (1995).

13. J. J. de Swart, Rev. Mod. Phys. 35, 916 (1963).

14. W. Dür and J. I. Cirac, "Multiparticle entanglement and its experimental detection," quant-ph/0011025 (2000).

15. M. Gell-Mann and Y. Neeman, The Eightfold Way (Benjamin Inc., New York, 1964).

16. S. Hill and W. K. Wootters, "Entanglement of a pair of quantum bits," quant$\mathrm{ph} / 9703041$ (1997).

17. A. A. Kirillov, Bull. Am. Math. Soc. 36, 433 (1999).

18. M. Kus and K. Zyczkowski, "Geometry of entangled states," quant-ph/0006068 (Oct. 2000).

19. A. S. Landsberg, Phys. Rev. Lett. 69, 865 (1992).

20. K. S. Mallesh and N. Mukunda, Pramana - J. Phys. 49, 371 (1997).

21. M. A. Nielsen, Phys. Rev. Lett. 83, 436 (1999).

22. M. Reck, A. Zellinger, H. J. Bernstein and P. Bertani, Phys. Rev. Lett. 73, 58 (1994).

23. B. C. Sanders, H. de Guise, S. D. Bartlett and W. Zhang, Phys. Rev. Lett. 86, 369 (2001).

24. B. C. Sanders, H. de Guise, D. J. Rowe and A. Mann, J. Phys. A: Math. Gen. 32, 7791 (1999).

25. J. Shapere and F. Wilczek, Geometric Phases in Physics (World Scientific, 1989), is the best available and comprehensive introduction and reprint volume on the subject.

26. P. B. Slater, "Bures geometry of three-level quantum systems," quant-ph/0008069 (2000).

27. P. B. Slater, "Increased efficiency of quantum state estimation using non-separable measurements," quant-ph/0006009 (2000).

28. J. Schlienz and G. Mahler, Phys. Rev. A52, 4396 (1995).

29. P. Zanardi and M. Rasetti, Phys. Lett. A264, 94 (1999). 